\title{
Article
}

\section{Probabilistic Three-Party Sharing of Operation on a Remote Qubit}

\author{
Chuanmei Xie ${ }^{1}$, Yimin Liu ${ }^{2}$, Hang Xing ${ }^{1}$ and Zhanjun Zhang ${ }^{1, *}$ \\ ${ }^{1}$ School of Physics \& Material Science, Anhui University, Hefei 230039, China; \\ E-Mails: cmxie@ahu.edu.cn (C.X.); xinghang1990hx@163.com (H.X.) \\ ${ }^{2}$ Department of Physics, Shaoguan University, Shaoguan 512005, China; \\ E-Mail: 1ym84111766@163.com
}

* Author to whom correspondence should be addressed; E-Mail: zjzhang@ahu.edu.cn.

Academic Editors: Demosthenes Ellinas, Giorgio Kaniadakis, Jiannis Pachos and Antonio M. Scarfone

Received: 29 October 2014 / Accepted: 27 January 2015 / Published: 12 February 2015

\begin{abstract}
A probabilistic tripartite single-qubit operation sharing scheme is put forward by utilizing a two-qubit and a three-qubit non-maximally entangled state as quantum channels. Some specific comparisons between our scheme and another probabilistic scheme are made. It is found that, if the product of the two minimal coefficients characterizing channel entanglements is greater than 3/16, our scheme is more superior than the other one. Nonetheless, the price is that more classical and quantum resources are consumed, and the operation difficulty is rather increased. Moreover, some important features of the scheme, such as its security, probability and sharer symmetry, are revealed through concrete discussions. Additionally, the experimental feasibility of our scheme is analyzed and subsequently confirmed according to the current experimental techniques.
\end{abstract}

Keywords: single-qubit operation sharing; non-maximally entangled state; probability; symmetry; security; experimental feasibility

PACS classifications: 03.65.Ta; 03.67.-a

\section{Introduction}

Shared quantum entanglements as important quantum resources and play key roles in the field of quantum information science, such as quantum key distribution [1-4], quantum state teleportation [5-9], 
quantum state sharing [10-13], quantum operation teleportation [14-16], and so on. Enlightened by the generalization of quantum state teleportation to quantum state sharing, with shared entanglements Zhang and Cheung [17] presented a new kind of quantum control named quantum operation sharing (QOS) in 2011. The QOS task in the simplest case can be depicted as follows. In a three-participant quantum scenario, an initial performer of a single-qubit operation wants to perform the single-qubit operation on a target state in a remote qubit in agents' site with the aid of his/her two agents. To fulfill such a task, they can make full use of local operations and classical communications as well as shared entanglements. Nonetheless, the initial performer trusts neither agent but only their entity. Because of this, the initial performer must assure the operation to be finally accomplished only via the two agents' collaboration. To some extent, QOS can be viewed as a generalization of quantum operation teleportation from two aspects of participant and security. In quantum operation teleportation, there is only one sender and one receiver, while in QOS the receiver is generalized to multi sharers. Moreover, in the former, no special security is demanded, while in the latter, a specific security against any inside sharers' cheating or outsiders' attack is required. Actually, QOS can be reduced to quantum operation teleportation if all agents are regarded as one. Hence, like quantum operation teleportation, QOS is also a kind of quantum remote control (encryption, decryption or destruction) on quantum information. In the intending quantum networks, it might play a very important role in activating some performances in life, such as missile emissions, quantum collective seal or unseam, remote joint destruction of quantum money, etc. Due to its potential important applications, QOS has attracted much attention so far [18-24].

It is worth pointing out that maximally entangled states are employed as quantum channels in the majority of existing QOS schemes. However, in a realistic situation, it is not currently possible to have a maximally entangled state at one's disposal. The decoherence and noise effect are usually unavoidable because of the interaction from the environment. Therefore, it is quite possible that quantum channels are disturbed and become non-maximally entangled states in practice though they might be initially prepared as maximally entangled ones. Such a decoherence problem can be mitigated but cannot be completely overcome easily. Consequently, it is necessary to consider how to utilize some non-maximally entangled states to fulfill some quantum tasks. Moreover, if there exist only some non-maximally entangled states, then one should make full use of them to realize QOS instead of doing nothing, particularly in some urgent or passive conditions. Given that the situation mentioned above does exist, utilizing non-maximally entangled states as quantum channels to fulfill the QOS task should be treated as an alternative scheme. Hence, it is of special importance and significance to study the implementation of some quantum tasks with non-maximally entangled states as quantum channels. As a matter of fact, some researchers have already utilized non-maximally entangled states to treat some quantum tasks in different quantum scenarios. For example, in 2002, Agrawal and Pati [9] first proposed a probabilistic quantum teleportation protocol, which is applicable for teleporting an arbitrary single-qubit state with a two-qubit non-maximally entnagled states; Gordon and Pigolin [25] presented two quantum state sharing protocols by taking three-qubit and two-qubit non-maximally entangled states as quantum channels in 2006, and so on. In this paper, we will propose a tripartite QOS scheme by using a two-qubit and a three-qubit non-maximally entangled state as quantum channels.

The rest of this paper is organized as follows. In Section 2, using a two-qubit and a three-qubit non-maximally entangled state, we will concretely present a three-party probabilistic QOS scheme. 
In Section 3, we will compare our scheme with that probabilistic scheme in [17] from the four aspects: the classical and quantum resource consumption, the difficulty or intensity of necessary operations, the success probability and the intrinsic efficiency of the schemes. Furthermore, we will discuss our scheme, including the scheme security, scheme probability, sharer symmetry and its experimental feasibility problem. Finally, we will make a concise summary in Section 4.

\section{Probabilistic Tripartite Scheme for Remotely Sharing Single-Qubit Operation}

In this section, we will put forward a probabilistic tripartite QOS scheme by utilizing a set of non-maximally entangled states. Before presenting the scheme, let us briefly depict the quantum task. In the scheme there are three legitimate participants, say Alice, Bob and Charlie. The latter two are Alice's distant agents. Alice needs to operate a state $|\varphi\rangle$ in a qubit in an agent's position via the concerned operation $U$. Without loss of generality, suppose the target qubit $b^{\prime}$ in the state $|\varphi\rangle$ is at Bob's site at the beginning, and its state is generally written as

$$
|\varphi\rangle_{b^{\prime}}=(\alpha|0\rangle+\beta|1\rangle)_{b^{\prime}}
$$

where $\alpha$ and $\beta$ are complex and satisfy $|\alpha|^{2}+|\beta|^{2}=1$. The state $U|\varphi\rangle=\left|\varphi^{\prime}\right\rangle$ is referred to as the conceivable state later. To fulfill the quantum task, Alice demands her two agents to help her and to collaborate with each other. Nevertheless, she is not willing to let the concerned operation be finally fulfilled by either agent solely, as she trusts neither of them but their entity. Hence, she must assure the operation to be implemented if and only if her agents cooperate. Taking advantage of the shared entanglements and local operation as well as classical communication, Alice and her agents can fulfill the quantum task as follows. The schematic demonstration is illustrated in Figure 1.
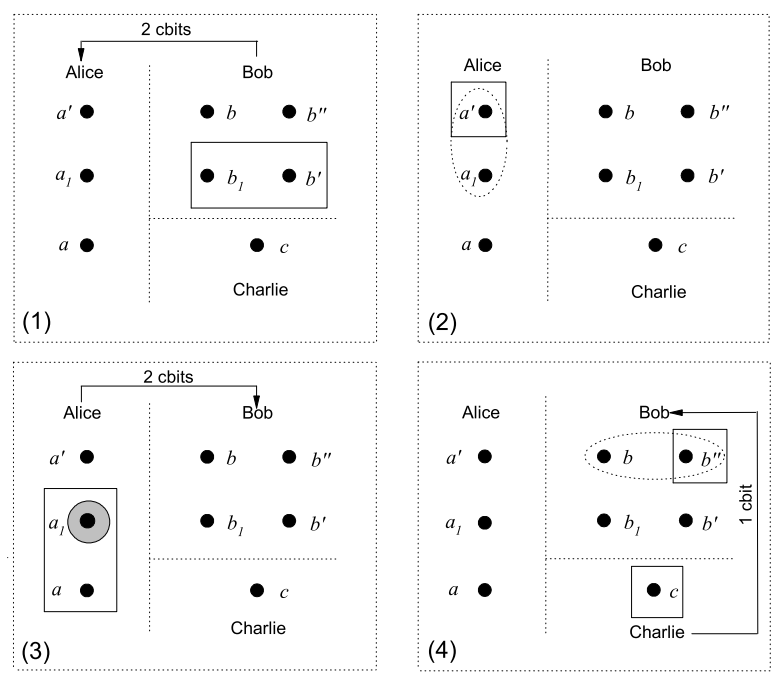

Figure 1. Illustration of the tripartite scheme for probabilisticslly sharing any arbitrary single-qubit operation with a two-qubit and a three-qubit non-maximally entangled states. Solid lines with arrows stand for the classical-message transfer. Solid dots denote qubits $a_{1}, a, a^{\prime}, b_{1}, b, b^{\prime}, b^{\prime \prime}$ and $c$, respectively. Solid squares and solid rectangles represent single-qubit and Bell-state measurements, respectively. The grey circle lables the concerned operation $U$ to be shared. Dotted ellipses label two-qubit unitary operations. The operations on any qubit are arranged from inner to outer. 
The entangled states they shared consist of a two-qubit and a three-qubit non-maximally states:

$$
|\mathcal{B}\rangle_{a_{1} b_{1}}=(\sqrt{1-n}|00\rangle+\sqrt{n}|11\rangle)_{a_{1} b_{1}}, \quad|\mathcal{G}\rangle_{a b c}=(\sqrt{1-m}|000\rangle+\sqrt{m}|111\rangle)_{a b c}
$$

where $m$ and $n$ are two known parameters and $0<m<1 / 2,0<n<1 / 2$, and the subindices denote the qubits securely distributed among the three users. Alice owns the qubit pair $\left(a_{1}, a\right)$, Bob has the pair $\left(b_{1}, b\right)$, and Charlie possesses the qubit $c$, respectively. After the preparation, they can do as follows:

\section{Bob starts to operate. [See Figure 1(1)]}

Bob performs a Bell-state measurement on his qubit pair $\left(b_{1}, b^{\prime}\right)$. Complementally, throughout this paper four Bell states are defined as:

$$
\left|\psi^{ \pm}\right\rangle_{a_{1} b_{1}}=\frac{1}{\sqrt{2}}(|00\rangle \pm|11\rangle)_{a_{1} b_{1}}, \quad\left|\phi^{ \pm}\right\rangle_{a_{1} b_{1}}=\frac{1}{\sqrt{2}}(|01\rangle \pm|10\rangle)_{a_{1} b_{1}}
$$

The state of qubits $a_{1}, b_{1}$ and $b^{\prime}$ is initially in

$$
|\mathcal{T}\rangle_{a_{1} b_{1} b^{\prime}}=|\mathcal{B}\rangle_{a_{1} b_{1}} \otimes|\varphi\rangle_{b^{\prime}}
$$

By virtue of the Bell state bases, it can be expanded as:

$$
|\mathcal{T}\rangle_{a b b^{\prime}}=\frac{1}{\sqrt{2}}\left[\left|\psi^{+}\right\rangle_{b_{1} b^{\prime}}\left|\chi_{00}\right\rangle_{a_{1}}+\left|\psi^{-}\right\rangle_{b_{1} b^{\prime}}\left|\chi_{01}\right\rangle_{a_{1}}+\left|\phi^{+}\right\rangle_{b_{1} b^{\prime}}\left|\chi_{10}\right\rangle_{a_{1}}+\left|\phi^{-}\right\rangle_{b_{1} b^{\prime}}\left|\chi_{11}\right\rangle_{a_{1}}\right]
$$

where

$$
\left.\begin{array}{rlrl}
\left|\chi_{00}\right\rangle_{a_{1}} & =(\sqrt{1-n} \alpha|0\rangle+\sqrt{n} \beta|1\rangle)_{a_{1}}, & \left|\chi_{01}\right\rangle_{a_{1}}=(\sqrt{1-n} \alpha|0\rangle-\sqrt{n} \beta|1\rangle)_{a_{1}}, \\
\left|\chi_{10}\right\rangle_{a_{1}}=(\sqrt{1-n} \beta|0\rangle+\sqrt{n} \alpha|1\rangle)_{a_{1}}, & \left|\chi_{11}\right\rangle_{a_{1}}=(\sqrt{1-n} \beta|0\rangle-\sqrt{n} \alpha|1\rangle)_{a_{1}} .
\end{array}\right\} .
$$

Easily one can see that Bob's measurements result in one of the following collapses (unnormalized):

$$
\left|\psi^{+}\right\rangle_{b_{1} b^{\prime}} \Rightarrow\left|\chi_{00}\right\rangle_{a_{1}}, \quad\left|\psi^{-}\right\rangle_{b_{1} b^{\prime}} \Rightarrow\left|\chi_{01}\right\rangle_{a_{1}}, \quad\left|\phi^{+}\right\rangle_{b_{1} b^{\prime}} \Rightarrow\left|\chi_{10}\right\rangle_{a_{1}}, \quad\left|\phi^{-}\right\rangle_{b_{1} b^{\prime}} \Rightarrow\left|\chi_{11}\right\rangle_{a_{1}}
$$

For example, if Bob gets $\left|\psi^{+}\right\rangle_{b_{1} b^{\prime}}$, the state of qubit $a_{1}$ has collapsed to the state $\left|\chi_{00}\right\rangle_{a_{1}}$. Similarly, other three measurement results induce other three collapses, respectively. See Formula (7). The occurrence probabilities of different collapses can be easily worked out. For $\left|\psi^{+}\right\rangle_{b_{1} b^{\prime}}$, it is

$$
P={ }_{a_{1} b_{1} b^{\prime}}\left\langle\mathcal{T}\left|I_{a_{1}} \otimes M_{b_{1} b^{\prime}}^{\dagger} M_{b_{1} b^{\prime}} \otimes I_{a_{1}}\right| \mathcal{T}\right\rangle_{a_{1} b_{1} b^{\prime}}=\frac{(1-n)|\alpha|^{2}+n|\beta|^{2}}{2}
$$

where $M=\left|\psi^{+}\right\rangle\left\langle\psi^{+}\right|$and $I=|0\rangle\langle 0|+| 1\rangle\langle 1|$. Analogously, probabilities of other outcomes $\left|\psi^{-}\right\rangle$, $\left|\phi^{+}\right\rangle$and $\left|\phi^{-}\right\rangle$are $\left[(1-n)|\alpha|^{2}+n|\beta|^{2}\right] / 2,\left[(1-n)|\beta|^{2}+n|\alpha|^{2}\right] / 2$ and $\left[(1-n)|\beta|^{2}+n|\alpha|^{2}\right] / 2$, respectively. Obviously, both $\left|\psi^{+}\right\rangle$and $\left|\psi^{-}\right\rangle$lead to the same probability and $\left|\phi^{+}\right\rangle$and $\left|\psi^{-}\right\rangle$lead to the same probability but different to the previous one. Toward the final goal of QOS task, Bob further notifies Alice of his measurement result by sending two classical-bit (cbit) messages. Complementarily, as a priori agreement in the scheme, the four Bell states $\left|\psi^{+}\right\rangle,\left|\psi^{-}\right\rangle,\left|\phi^{+}\right\rangle$and $\left|\phi^{-}\right\rangle$correspond to the two cbit messages $(0,0),(0,1),(1,0)$ and $(1,1)$, respectively. 
II. After receiving Bob's message, Alice does as follows. [See Figure 1(2-3)]

First, Alice incorporates an auxiliary qubit $a^{\prime}$ in the state $|0\rangle$ to form a pair $\left(a_{1}, a^{\prime}\right)$, and performs a peculiar two-qubit operation on the pair according to the two cbit messages Bob has promulgated. Specifically, she operates $V^{(j k)}(n)$ on the composite state $\left|\chi_{j k}\right\rangle_{a_{1}}|0\rangle_{a^{\prime}}$, where $V^{(j k)}(n)$ in the ordering bases $\{|00\rangle,|01\rangle,|10\rangle,|11\rangle\}$ take the following explicit matric forms

$$
\begin{aligned}
V^{(00)}(n) & =\left(\begin{array}{cccc}
\sqrt{\frac{n}{1-n}} & \sqrt{\frac{1-2 n}{1-n}} & 0 & 0 \\
0 & 0 & 0 & -1 \\
0 & 0 & 1 & 0 \\
\sqrt{\frac{1-2 n}{1-n}} & -\sqrt{\frac{n}{1-n}} & 0 & 0
\end{array}\right), V^{(01)}(n)=\left(\begin{array}{cccc}
\sqrt{\frac{n}{1-n}} & \sqrt{\frac{1-2 n}{1-n}} & 0 & 0 \\
0 & 0 & 0 & 1 \\
0 & 0 & -1 & 0 \\
\sqrt{\frac{1-2 n}{1-n}} & -\sqrt{\frac{n}{1-n}} & 0 & 0
\end{array}\right), \\
V^{(10)}(n) & =\left(\begin{array}{cccc}
\sqrt{\frac{1-2 n}{1-n}} & \sqrt{\frac{n}{1-n}} & 0 & 0 \\
0 & 0 & 1 & 0 \\
0 & 0 & 0 & -1 \\
\sqrt{\frac{n}{1-n}} & -\sqrt{\frac{1-2 n}{1-n}} & 0 & 0
\end{array}\right), V^{(11)}(n)=\left(\begin{array}{cccc}
\sqrt{\frac{1-2 n}{1-n}} & \sqrt{\frac{n}{1-n}} & 0 & 0 \\
0 & 0 & -1 & 0 \\
0 & 0 & 0 & 1 \\
\sqrt{\frac{n}{1-n}} & -\sqrt{\frac{1-2 n}{1-n}} & 0 & 0
\end{array}\right) .
\end{aligned}
$$

Alice's operation entangles the qubit pair and makes the state evolve to one of the following states:

$$
\left.\begin{array}{c}
V_{a_{1} a^{\prime}}^{(00)}(n)\left|\chi_{00}\right\rangle_{a_{1}}|0\rangle_{a^{\prime}}=\sqrt{n}|\varphi\rangle_{a_{1}}|0\rangle_{a^{\prime}}+\sqrt{1-2 n} \alpha|1\rangle_{a_{1}}|1\rangle_{a^{\prime}}, \\
V_{a_{1} a^{\prime}}^{(01)}(n)\left|\chi_{01}\right\rangle_{a_{1}}|0\rangle_{a^{\prime}}=\sqrt{n}|\varphi\rangle_{a_{1}}|0\rangle_{a^{\prime}}+\sqrt{1-2 n} \alpha|1\rangle_{a_{1}}|1\rangle_{a^{\prime}}, \\
V_{a_{1} a^{\prime}}^{(10)}(n)\left|\chi_{10}\right\rangle_{a_{1}}|0\rangle_{a^{\prime}}=\sqrt{n}|\varphi\rangle_{a_{1}}|1\rangle_{a^{\prime}}+\sqrt{1-2 n} \beta|0\rangle_{a_{1}}|0\rangle_{a^{\prime}}, \\
V_{a_{1} a^{\prime}}^{(11)}(n)\left|\chi_{10}\right\rangle_{a_{1}}|0\rangle_{a^{\prime}}=\sqrt{n}|\varphi\rangle_{a_{1}}|1\rangle_{a^{\prime}}+\sqrt{1-2 n} \beta|0\rangle_{a_{1}}|0\rangle_{a^{\prime}}
\end{array}\right\}
$$

Second, Alice measures the ancilla $a^{\prime}$ with the bases $\{|0\rangle,|1\rangle\}$. Obviously, in the case that Alice received the two cbit messages $(0,0)$ or $(0,1)$ from $\mathrm{Bob}$, if she gets $|0\rangle_{a^{\prime}}$, the initial quantum state $|\varphi\rangle$ is recovered in her qubit $a_{1}$, and the success probability is $n /\left[(1-n)|\alpha|^{2}+n|\beta|^{2}\right]$. Otherwise, the initial state is destroyed and accordingly the teleportation fails. As for the other case Alice got the messages $(1,0)$ or $(1,1)$, the initial state will be rebuilt in her qubit $a_{1}$ as long as her measurement out is $|1\rangle$. In this case, the success probability is $n /\left[(1-n)|\beta|^{2}+n|\alpha|^{2}\right]$. Consequently, the total success probability of the teleportation is $2 n$.

Thirdly, Alice performs the concerned operation $U$ on her qubit $a_{1}$, i.e., $U_{a_{1}}|\varphi\rangle_{a_{1}} \equiv\left|\varphi^{\prime}\right\rangle_{a_{1}} \equiv$ $\left(\alpha^{\prime}|0\rangle+\beta^{\prime}|1\rangle\right)_{a_{1}}$. Note that, at this moment the conceivable state $U_{a_{1}}|\varphi\rangle_{a_{1}}$ has been constructed, but in Alice's qubit $a_{1}$. It is obviously not the final goal of QOS. The final goal is to perform the concerned operation $U$ on the target state $|\varphi\rangle$ in the qubit located at remote agents' site. Hence, after this process further treatments are still needed in order to accomplish the task.

Finally, Alice measures her qubit pair $\left(a_{1}, a\right)$ with the Bell state bases and announces her measurement result to Bob in terms of the priori agreement. It is known that the state of the total system consisting of the qubit quartet $\left(a_{1}, a, b, c\right)$ reads:

$$
|\mathcal{Q}\rangle_{a_{1} a b c}=\left|\varphi^{\prime}\right\rangle_{a_{1}} \otimes|\mathcal{G}\rangle_{a b c}
$$


It can be rewritten as:

$$
\begin{aligned}
|\mathcal{Q}\rangle_{a_{1} a b c}= & \frac{1}{\sqrt{2}}\left[\left|\psi^{+}\right\rangle_{a_{1} a}\left(\sqrt{1-m} \alpha^{\prime}|00\rangle+\sqrt{m} \beta^{\prime}|11\rangle\right)_{b c}\right. \\
& +\left|\psi^{-}\right\rangle_{a_{1} a}\left(\sqrt{1-m} \alpha^{\prime}|00\rangle-\sqrt{m} \beta^{\prime}|11\rangle\right)_{b c} \\
& +\left|\phi^{+}\right\rangle_{a_{1} a}\left(\sqrt{m} \alpha^{\prime}|11\rangle+\sqrt{1-m} \beta^{\prime}|00\rangle\right)_{b c} \\
& \left.+\left|\phi^{-}\right\rangle_{a_{1} a}\left(\sqrt{m} \alpha^{\prime}|11\rangle-\sqrt{1-m} \beta^{\prime}|00\rangle\right)_{b c}\right]
\end{aligned}
$$

Obviously, Alice's measurements collapse the state in Bob's and Charlie's qubits. Specifically,

$$
\left.\begin{array}{rl}
\left|\psi^{+}\right\rangle_{a_{1} a} & \Longrightarrow\left|\mathcal{O}_{00}\right\rangle_{b c}=\left(\sqrt{1-m} \alpha^{\prime}|00\rangle+\sqrt{m} \beta^{\prime}|11\rangle\right)_{b c}, \\
\left|\psi^{-}\right\rangle_{a_{1} a} & \Longrightarrow\left|\mathcal{O}_{01}\right\rangle_{b c}=\left(\sqrt{1-m} \alpha^{\prime}|00\rangle-\sqrt{m} \beta^{\prime}|11\rangle\right)_{b c}, \\
\left|\phi^{+}\right\rangle_{a_{1} a} & \Longrightarrow\left|\mathcal{O}_{10}\right\rangle_{b c}=\left(\sqrt{m} \alpha^{\prime}|11\rangle+\sqrt{1-m} \beta^{\prime}|00\rangle\right)_{b c}, \\
\left|\phi^{-}\right\rangle_{a_{1} a} & \Longrightarrow\left|\mathcal{O}_{11}\right\rangle_{b c}=\left(\sqrt{m} \alpha^{\prime}|11\rangle-\sqrt{1-m} \beta^{\prime}|00\rangle\right)_{b c} .
\end{array}\right\}
$$

For the probability of getting $\left|\psi^{+}\right\rangle_{a_{1} a}$, it is

$$
P={ }_{a_{1} a b c}\left\langle\mathcal{Q}\left|\mathcal{I}_{b c} \otimes M_{a_{1} a}^{\dagger} M_{a_{1} a} \otimes \mathcal{I}_{b c}\right| \mathcal{Q}\right\rangle_{a_{1} a b c}=\frac{(1-m)\left|\alpha^{\prime}\right|^{2}+m\left|\beta^{\prime}\right|^{2}}{2}
$$

where $\mathcal{I}=|00\rangle\langle 00|+| 01\rangle\langle 01|+| 10\rangle\langle 10|+| 11\rangle\langle 11|$. Similarly, the probability of getting $\left|\psi^{-}\right\rangle_{a_{1} a}$ is the same as the probability of getting $\left|\psi^{+}\right\rangle_{a_{1} a}$. For the probabilities of getting $\left|\phi^{+}\right\rangle_{a_{1} a}$ and getting $\left|\phi^{-}\right\rangle_{a_{1} a}$, they are same and are $\left[(1-m)\left|\beta^{\prime}\right|^{2}+m\left|\alpha^{\prime}\right|^{2}\right] / 2$.

\section{Bob and Charlie collaborate to reconstruct the conceivable state. [See Figure 1(4)]}

In this stage, if Bob and Charlie collaborate and decide Bob to conclusively fulfill the reconstruction, then they can do as follows:

Charlie measures his qubit $c$ with the bases $\{|+\rangle,|-\rangle\}$. In this paper, $| \pm\rangle \equiv(|0\rangle \pm|1\rangle) / \sqrt{2}$ is defined. Then he informs Bob of his measurement outcome via a classical channel. In the priori agreement, the single cbit message "0" ("1") corresponds the state $|+\rangle_{c}\left(|-\rangle_{c}\right)$. The four possible state in Bob's and Charlie's qubits can be written as:

$$
\left.\begin{array}{l}
\left|\mathcal{O}_{00}\right\rangle_{b c}=\frac{1}{\sqrt{2}}\left[\left(\sqrt{1-m} \alpha^{\prime}|0\rangle+\sqrt{m} \beta^{\prime}|1\rangle\right)_{b}|+\rangle_{c}+\left(\sqrt{1-m} \alpha^{\prime}|0\rangle-\sqrt{m} \beta^{\prime}|1\rangle\right)_{b}|-\rangle_{c}\right], \\
\left|\mathcal{O}_{01}\right\rangle_{b c}=\frac{1}{\sqrt{2}}\left[\left(\sqrt{1-m} \alpha^{\prime}|0\rangle-\sqrt{m} \beta^{\prime}|1\rangle\right)_{b}|+\rangle_{c}+\left(\sqrt{1-m} \alpha^{\prime}|0\rangle+\sqrt{m} \beta^{\prime}|1\rangle\right)_{b}|-\rangle_{c}\right], \\
\left|\mathcal{O}_{10}\right\rangle_{b c}=\frac{1}{\sqrt{2}}\left[\left(\sqrt{m} \alpha^{\prime}|1\rangle+\sqrt{1-m} \beta^{\prime}|0\rangle\right)_{b}|+\rangle_{c}+\left(\sqrt{m} \alpha^{\prime}|1\rangle-\sqrt{1-m} \beta^{\prime}|0\rangle\right)_{b}|-\rangle_{c}\right], \\
\left|\mathcal{O}_{11}\right\rangle_{b c}=\frac{1}{\sqrt{2}}\left[\left(\sqrt{m} \alpha^{\prime}|1\rangle-\sqrt{1-m} \beta^{\prime}|0\rangle\right)_{b}|+\rangle_{c}+\left(\sqrt{m} \alpha^{\prime}|1\rangle+\sqrt{1-m} \beta^{\prime}|0\rangle\right)_{b}|-\rangle_{c}\right] .
\end{array}\right\} .
$$

Upon receiving Charlie's message, Bob firstly introduces an auxiliary qubit $b^{\prime \prime}$ in the state $|0\rangle$, and then carries out a peculia two-qubit operation on the qubit pair $\left(b, b^{\prime \prime}\right)$ according to Alice's and Charlie's messages. At last, he measures his qubit $b^{\prime \prime}$ with the bases $\{|0\rangle,|1\rangle\}$. For example, if Bob receives the message $(0,0)$ from Alice and " 1 " from Charlie, he executes $V^{(01)}(m)$ on the qubit pair $\left(b, b^{\prime \prime}\right)$. The operation transforms the state of qubit pair $\left(b, b^{\prime \prime}\right)$ to

$$
V_{b b^{\prime \prime}}^{(01)}(m)\left(\sqrt{1-m} \alpha^{\prime}|0\rangle-\sqrt{m} \beta^{\prime}|1\rangle\right)_{b}|0\rangle_{b^{\prime \prime}}=\sqrt{m}|\varphi\rangle_{b}|0\rangle_{b^{\prime \prime}}+\sqrt{1-2 m} \alpha^{\prime}|1\rangle_{b}|1\rangle_{b^{\prime \prime}}
$$

If Bob measures his qubit $b^{\prime \prime}$ and gets $|0\rangle$, the conceivable state is reconstructed in his qubit $b$, and the probability is $m /\left[(1-m)\left|\alpha^{\prime}\right|^{2}+m\left|\beta^{\prime}\right|^{2}\right]$. Otherwise, the conceivable state is destroyed and hence 
the sharing fails. As for other cases, the circumstance is almost similar and in each case Bob can deterministically get the conceivable state. In the state sharing process, one can easily work out the total probability $2 m$. Hence, for our present scheme the total success probability is $2 n \times 2 m=4 m n$.

\section{Comparisons and Discussions}

Now let us compare our scheme (referred to as XLXZ ) with a probabilistic scheme (called ZC) presented in Reference [17]. Before comparisons, we summarize the two schemes with respect to the following four aspects: the cost of quantum and classical resources, the difficulty or intensity of necessary operations, the scheme success probability, and the intrinsic efficiency. See Table 1 for details.

Table 1. Comparisons between schemes XLXZ and ZC. AQ: auxiliary quBit; QRC: quantum resource consumption; NO: necessary operations; CRC: classical resource consumption; BM: Bell-state measurement; SM: single-qubit measurement; TQUO: two-qubit unitary operation; SQUO: single-qubit unitary operation. The intrinsic efficiency there is difined as $\eta=P /\left(Q_{t}+C_{t}\right)$, where $Q_{t}$ is the number of the qubits used as quantum channels, $C_{t}$ is the classical bits transmitted via classical channels, and $P$ is the final success probability.

\begin{tabular}{cccccc}
\hline $\mathrm{S}$ & $\mathrm{QRC}$ & $\mathrm{CRC}$ & $\mathrm{NO}$ & $P$ & $\eta$ \\
\hline $\mathrm{XLXZ}$ & $|\mathcal{B}\rangle,|\mathcal{G}\rangle, 2 \mathrm{AQs}$ & 5 cbits & 2TQUOs, 2SMs, 2BMs & $4 m n$ & $m n / 3$ \\
$\mathrm{ZC}$ & $\left|\psi^{+}\right\rangle,\left|\psi^{+}\right\rangle$ & 4 cbits & 2SQUOs, TQUO, 2SMs, BM & $1 / 2$ & $1 / 16$ \\
\hline
\end{tabular}

From the second and third columns of the table, one can see that, XLXZ consumes a 2-qubit and a 3-qubit non-maximally entangled states as well as two auxiliary qubits, while ZC consumes two 2-qubit maximally entangled states. Very intuitively, the quantum resource consumption is reduced in ZC, the classical resource consumption in ZC is less than that in XLXZ. Further inspect the fourth column. Note that, Bell-state measurements can be decomposed an ordering combination of a two-qubit CNOT operation and a single-qubit Hadamard operation as well as two single-qubit measurements. Taking account of this decomposition, one can see that the operation difficulty in XLXZ is relatively higher than that in ZC. This indicates that ZC is better than XLXZ as far as the cost of quantum and classical resource consumptions as well as the operation difficulty are concerned. Nevertheless, inspecting the fifth columns of the table, one can see that, (1) the success probability in XLXZ is $4 m n$, which increases from 0 to 1 with the increasing $m$ and $n$; (2) the success probability in $\mathrm{ZC}$ is $1 / 2$. It is obvious that both schemes are probabilistic. The success probability in XLXZ is dependent on $m$ and $n$, while that in ZC is a certain value. If $m n>1 / 8$, then the success probability in XLXZ is bigger than that in ZC. In particular, when $m n=1 / 4$, that is, when these states are maximally entangled, the success probability in XLXZ reaches to 1, which is twice as higher as that in ZC scheme. It indicates that in this case our present scheme overwhelms ZC from the aspect of the success probability. Moreover, if $m n>3 / 16$, apart from the success probability, the intrinsic efficiency in XLXZ is bigger than that in ZC. Consider them comprehensively, one is readily to draw a conclusion that XLXZ is more superior than ZC if $m n$ is set to be $m n>3 / 16$. Of course, the cost is that more classical and quantum resources are consumed, and XLXZ has higher operation difficulty. 
Now let us make some brief discussions about the present scheme to show their important features. Obviously, our present scheme is a probabilistic one, and its probability is dependent on the parameters of the two non-maximally channels. In addition, in our scheme the conceivable state can be reconstructed at either Bob's or Charlie's site, which can be seen from Formula (14). This indicates that our scheme is symmetric as far as the shares are concerned. Hence, probability and symmetry are two features of our scheme. As for the security of the scheme, being a QOS scheme, its security should be assured in principle. Actually, we have assumed in priori that quantum channels are secure. They are preconditions of our scheme. As a matter of fact, the states taken as quantum channels are very similar to those in References [26-28] to some extent. Whether they are secure during the qubit distribution or not can be easily checked by using the mature sampling technique and any attacker's perturbation can be detected. They are applicable for our present QOS scheme, too. For simplicity, here we do not repeat them anymore. In this sense, we think our present scheme is secure. One the other hand, as mentioned just, our scheme is symmetric for sharers. Such a feature of sharer symmetry relative to the uncertainty of choosing the starter and the final constructor can enhance the scheme security to some extent.

At last, let us simply discuss the experimental feasibility of our scheme. In our scheme, a two-qubit and a three-qubit non-maximally entangled states are employed as quantum channels. During the whole sharing process only Bell-state, single-qubit measurements and two-qubit unitary operations are needed and all of them are locally performed. To our knowledge, it has been reported that the Bell-state measurements, the single-qubit measurements and two-qubit unitary operations have already been realized in many quantum systems [29-34] by far. Therefore, our present probabilistic three-party scheme is thoroughly feasible with respect to the current experimental technologies.

\section{Summary}

In this paper we have presented a three-party QOS scheme by using two non-maximally entangled states as quantum channels. The scheme is applicable for probabilistically sharing an arbitrary single-qubit operation on a remote qubit in any sharer's position. After presenting the scheme, we have compared the scheme with the ZC scheme [17]. It is found that in the case that $m n>3 / 16$, the success probability and the intrinsic efficiency in the present scheme are bigger than that in the ZC scheme. The price the present scheme needs to pay is to consume more quantum and classical resources and to have a little higher operation difficulty. Through concrete discussions, we have further revealed some important features of our scheme, such as its security, probability and sharer symmetry. Finally, we have simply demonstrated the experimental feasibility of our scheme in terms of current techniques.

\section{Acknowledgments}

This work was supported by the National Natural Science Foundation of China (NNSFC) under Grant Nos. 11375011 and 11372122, the Natural Science Foundation of Anhui province under Grant No. 1408085MA12, and the 211 Project of Anhui University. 


\section{Author Contributions}

Zhanjun Zhang conceived the present theoretical frame; All authors contributed to write the paper. All authors have read and approved the final manuscript.

\section{Conflicts of Interest}

The authors declare no conflict of interest.

\section{References}

1. Ekert, A.K. Quantum cryptography based on Bell's theorem. Phys. Rev. Lett. 1991, 67, 661-663.

2. Bennett, C.H. Quantum cryptography using any two nonorthogonal states. Phys. Rev. Lett. 1992, $68,3121-3124$.

3. Bennett, C.H.; Brassard, G.; Mermin, N.D. Quantum cryptography without Bell's theorem. Phys. Rev. Lett. 1992, 68, doi:10.1103/PhysRevLett.68.557.

4. Deng, F.G.; Long, G.L.; Liu, X.S. Two-step quantum direct communication protocol using the Einstein-Podolsky-Rosen pair block. Phys. Rev. A 2003, 68, doi:10.1103/PhysRevA.68.042317.

5. Bennett, C.H.; Brassard, G.; Crépeau, C.; Jozsa, R.; Peres, A.; Wootters, W.K. Teleporting an unknown quantum state via dual classical and Einstein-Podolsky-Rosen channels. Phys. Rev. Lett. 1993, 70, 1895-1899.

6. Bouwmeester, D.; Pan, J.-W.; Mattle, K.; Eibl, M.; Weinfurter, H.; Zeilinger, A. Experimental quantum teleportation. Nature 1997, 390, 575-579.

7. Furusawa, A.; Sørensen, J.L.; Braunstein, S.L.; Fuchs, C.A.; Kimble, H.J.; Polzik, E.S. Unconditional quantum teleportation. Science 1998, 282, 706-709.

8. Muralidharan, S.; Panigrahi, P.K. Perfect teleportation, quantum-state sharing, and superdense coding through a genuinely entangled five-qubit state. Phys. Rev. A 2008, 77, doi:10.1103/ PhysRevA.77.032321

9. Agrawal, P.; Pati, A.K. Probabilistic quantum teleportation. Phys. Lett. A 2002, 305, 12-17.

10. Hillery, M.; Bužek, V.; Berthiaume, A. Quantum secret sharing. Phys. Rev. A 1999, 59, 1829-1834.

11. Paul, N.; Menon, J.V.; Karumanchi, S.; Muralidharan, S; Panigrahi, P.K. Quantum tasks using six qubit cluster states. Quantum Inf. Process. 2011, 10, 619-632.

12. Muralidharan, S.; Jain, S.; Panigrahi, P.K. Spitting of quantum information using $N$-qubit linear cluster states. Opt. Commun. 2011, 284, 1082-1085.

13. Muralidharan, S.; Panigrahi, P.K. Quantum-information splitting using multipartite cluster states. Phys. Rev. A 2008, 78, doi:10.1103/PhysRevA.78.062333.

14. Huelga, S.F.; Vaccaro, J.A.; Chefles, A. Quantum remote control: Teleportation of unitary operations. Phys. Rev. A 2001, 63, doi:10.1103/PhysRevA.63.042303.

15. Huelga, S.F.; Plenio, M.B.; Vaccaro, J.A. Remote control of restricted sets of operations: Teleportation of angles. Phys. Rev. A 2002, 65, doi:10.1103/PhysRevA.65.042316.

16. Dür, W.; Vidal, G.; Cirac, J.I. Optimal conversion of nonlocal unitary operations. Phys. Rev. Lett. 2002, 89, doi:10.1103/PhysRevLett.89.057901. 
17. Zhang, Z.J.; Cheung, C.Y. Shared quantum remote control: quantum operation sharing. J. Phys. $B$ 2011, 44, doi:10.1088/0953-4075/44/16/165508.

18. Ji, Q.B.; Liu, Y.M.; Yin, X.F.; Liu, X.S.; Zhang, Z.J. Quantum operation sharing with symmetric and asymmetric W states. Quantum Inf. Process. 2013, 12, 2453-2464.

19. Ji, Q.B.; Liu, Y.M.; Xie, C.M.; Yin, X.F.; Zhang, Z.J. Tripartite quantum operation sharing with two asymmetric three-qubit W states in five entanglement structures. Quantum Inf. Process. 2014, $13,1659-1676$.

20. Duan Y.J.; Zha, X.W. Remotely sharing a single-qubit operation via a six-qubit entangled state. Int. J. Theor. Phys. 2014, doi:10.1007/s10773-014-2283-6.

21. Xing, H.; Liu, Y.; Xie, C.; Ji, Q.; Zhang, Z. Four-party deterministic operation sharing with six-qubit cluster state. Quantum Inf. Process. 2014, 13, 1553-1562.

22. Wang, S.F.; Liu, Y.; Chen, J.; Liu, X.; Zhang, Z. Deterministic single-qubit operation sharing with five-qubit cluster state. Quantum Inf. Process. 2013, 12, 2497-2507.

23. Liu, D.C.; Liu, Y.; Xie, C.; Yin, X.; Liu, X.; Zhang, Z. Shared quantum control via sharing operation on remote single qutrit. Quantum Inf. Process. 2013, 12, 3527-3542.

24. Ye, B.L.; Liu, Y.; Liu, X.-S.; Zhang, Z.-J. Remotely sharing a single-qubit operation with a five-qubit genuine state. Chin. Phys. Lett. 2013, 30, doi:10.1088/0256-307X/30/2/020301.

25. Gordon, G.; Rigolin, G. Generalized quantum-state sharing. Phys. Rev. A 2006, 73, doi:10.1103/ PhysRevA.73.062316.

26. Zhang, Z.J. Multiparty quantum secret sharing of secure direct communication. Phys. Lett. A 2005, 342, 60-66.

27. Deng, F.G.; Li, X.H.; Zhou, H.Y.; Zhang, Z.J. Improving the security of multiparty quantum secret sharing against Trojan horse attack. Phys. Rev. A 2005, 72, doi:10.1103/PhysRevA.72.044302.

28. Deng, F.G.; Zhou, H.Y.; Long, G.L. Bidirectional quantum secret sharing and secret splitting with polarized single photons. Phys. Lett. A 2005, 337, 329-334.

29. Riebe, M.; Häffner. H.; Roos, C.F.; Hänsel, W.; Benhelm, J.; Lancaster, G.P.T.; Körber, T.W.; Becher, C.; Schmidt-Kaler, F.; James, D.F.V.; et al. Deterministic quantum teleportation with atoms. Nature 2004, 429, 734-737.

30. Solano, E.; Cesar, C.L.; de Matos Filho, R.L.; Zagury, N. Reliable teleportation in trapped ions. Eur. Phys. J. D 2001, 13, 121-128.

31. Ikram, M.; Zhu, S.Y.; Zubairy, M.S. Quantum teleportation of an entangled state. Phys. Rev. A 2000, 62, doi:10.1103/PhysRevA.62.022307.

32. Zheng, S.B. Scheme for approximate conditional teleportation of an unknown atomic state without the Bell-state measurement. Phys. Rev. A 2004, 69, doi:10.1103/PhysRevA.69.064302.

33. Lim, H.T.; Kim, Y.S.; Ra, Y.S.; Bae, J.; Kim, Y.H. Experimental realization of an approximate transpose operation for qutrit systems using a structural physical approximation. Phys. Rev. A 2012, 86, doi:10.1103/PhysRevA.86.042334. 
34. Yang, C.P.; Su, Q.P.; Han, S.Y. Generation of Greenberger-Horne-Zeilinger entangled states of photons in multiple cavities via a superconducting qutrit or an atom through resonant interaction. Phys. Rev. A 2012, 86, doi:10.1103/PhysRevA.86.022329.

(c) 2015 by the authors; licensee MDPI, Basel, Switzerland. This article is an open access article distributed under the terms and conditions of the Creative Commons Attribution license (http://creativecommons.org/licenses/by/4.0/). 